\title{
La seconda vita delle immagini dall'archivio al web. Una proposta didattica
}

di Irene Zoppi



Ricerca sul web, lettura e riutilizzo critico di una fonte fotografica

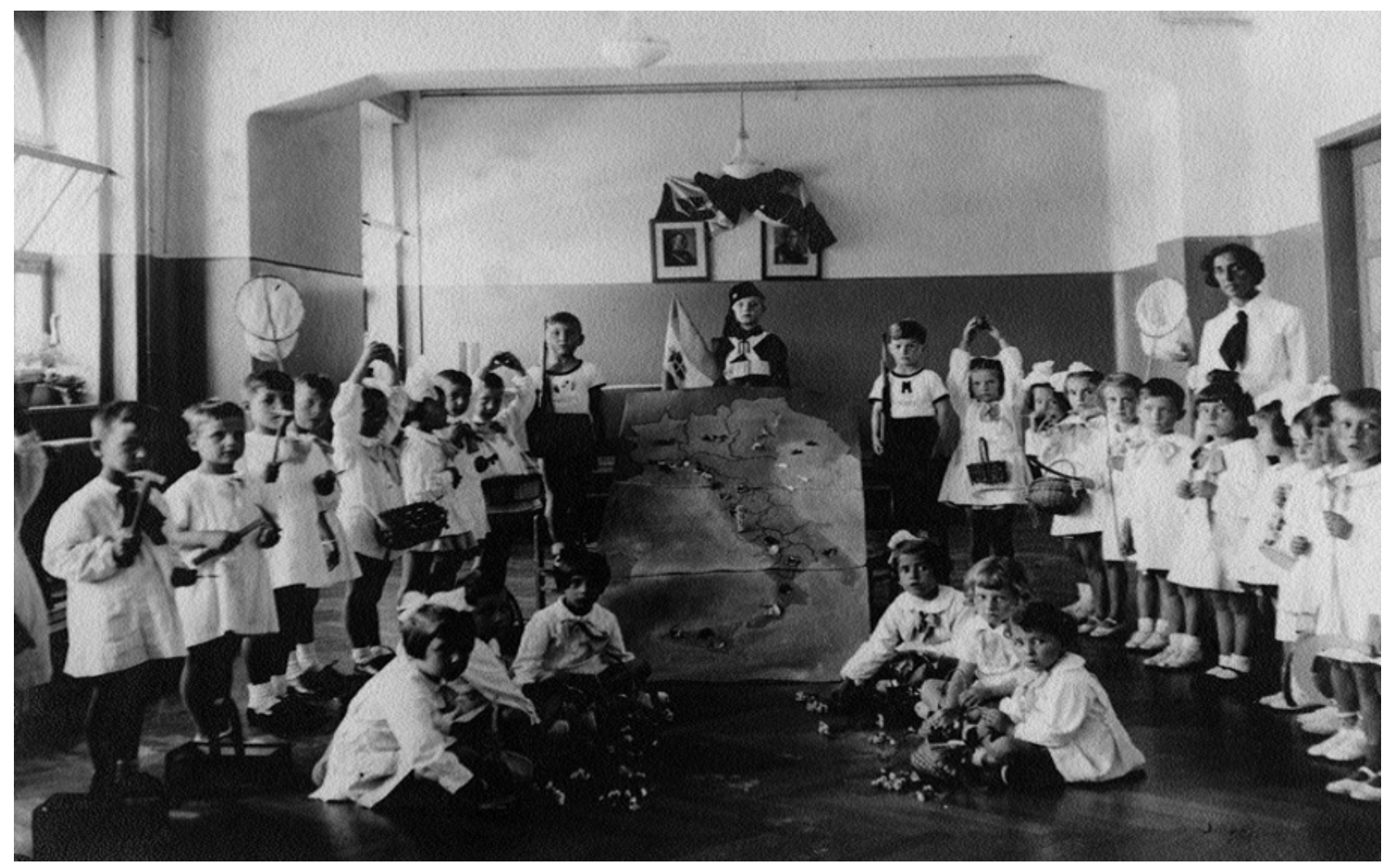

La fotografia proveniente dall'Archivio Storico Indire

\section{Abstract}

La ricerca e il corretto uso delle forme iconografiche sono importanti sia nella critica professionale delle fonti che nel curricolo scolastico. In particolare, le fotografie, oggi così diffuse e facilmente reperibili in internet, sono solo apparentemente oggetti di facile lettura. In questo articolo affrontiamo il problema della loro interpretazione in un contesto didattico. Quali sono le richieste cui viene sottoposto lo studente? Quali rischi si corrono utilizzando un approccio ingenuo verso questo tipo di materiale e verso la rete in cui è rintracciabile? Cerchiamo di rispondere a queste domande attraverso la ricognizione e l'analisi del riuso di alcune immagini dell'Archivio Storico Indire on line. Il lavoro costituisce una proposta didattica sulla lettura e l'uso delle

fonti. Comprende anche le possibili ricadute formative generali, lo sviluppo di competenze storiche e digitali, di cittadinanza attiva e di pensiero critico. 
Si può facilmente supporre che il web libero sia il primo e più immediato repertorio di consultazione per i giovani studenti di oggi. La rete, utilizzata normalmente per la ricerca, lo studio e l'approfondimento, sarà dunque anche il luogo in cui i ragazzi cercano e trovano le immagini utili a fornire riscontri visivi di concetti didattici, riutilizzabili in elaborati di vario genere: saggi, confronti, relazioni e presentazioni. Non è certo una pratica da demonizzare. La facilità di accesso che caratterizza internet ne fa uno strumento di lavoro molto potente che però deve essere analizzato nelle sue peculiarità e persino sfruttato per l'esercizio di ricerca critica delle fonti siano queste fotografiche, come qui proporremo, o di altra tipologia: iconografiche, testuali, orali etc.

La rete, se adeguatamente interrogata con i tools offerti dai motori di ricerca, ci permette, infatti, di rintracciare e confrontare le diverse provenienze delle immagini: nei database archivistici dei luoghi di conservazione e repository open access, o nelle molteplici tipologie di pubblicazioni diffuse nel web, rivelandosi in questo modo un utile strumento didattico.

Fare attenzione all'origine delle immagini che si cercano e riusano per corredare una presentazione o un testo qualsiasi, dovrebbe essere una pratica altrettanto richiesta e utile quanto la corretta citazione testuale o bibliografica. L'apparente facilità di lettura dell'immagine, in particolare di quella fotografica, invece, stimola spesso un uso approssimativo di questi documenti, non solo negli studenti delle scuole di ogni ordine e grado. Occorre invece trattarli correttamente come fonti: quali operazioni dobbiamo compiere per farlo? Come possiamo collocarli nel loro contesto originale, o almeno provarci? Quali domande dobbiamo porci per una corretta interpretazione delle immagini che liberamente e in gran quantità il web ci fornisce?

\section{La fotografia nel web libero e il suo utilizzo in didattica}

Nell'ambito di workshop per docenti e studenti realizzati dall'Archivio Storico Indire[1] (d'ora in poi ASI), è nata l'idea di sviluppare un «test» di ricerca in rete a partire da alcune fotografie dell'archivio, su cui costruire un'attività didattica riproponibile dai docenti in classe (gestibile in autonomia, grazie agli strumenti reperibili on line, e adattabile in quanto modulabile dal docente secondo gradi diversi di ricerca avanzata e difficoltà); l'obiettivo era stimolare l'analisi di una fotografia, rintracciarne i suoi molteplici riutilizzi nel web e quindi l'attribuzione di nuovi significati ai suoi contenuti.

Per strutturare l'attività abbiamo usato fotografie dell'ASI, in modo da fornire una risposta possibile alla provenienza della fotografia, ma la discussione è chiaramente applicabile ad ogni fonte, non solo fotografica. Tra le premesse evidenziamo di non poter essere del tutto certi che le immagini analizzate provengano proprio dall'ASI, istituto che non le ha prodotte; dagli anni Venti i suoi antecedenti istituzionali (Mostra della scuola 1925, Museo della scuola, Centro Didattico Nazionale) hanno acquisito le fotografie per donazione da parte degli istituti scolastici chiamati a inviarle, ricevendole già stampate, non accompagnate dal negativo, talvolta già montate su cartoncino o album e didascalizzate. Questi aspetti ci rendono coscienti che esse abbiano avuto altri contesti di creazione (scatto e negativo) e possibili manipolazioni e fruizioni, precedenti a quelle note dell'ASI[2], tuttavia nel contesto dell'attività, riferirsi ai dati archivistici disponibili è funzionale a orientare e fornire un riscontro attendibile o in parte tale.

Lo scopo della nostra proposta è anche sottolineare come il riutilizzo di un'immagine e il suo inserimento in nuovi ambiti comunicativi possa rispondere a esigenze di rilettura e interpretazione storica (benché nell'ottica di una corretta filologia), giornalistica (pur mantenendo una deontologia), $\mathrm{o}$ anche letteraria $\mathrm{o}$ artistica... Sta al fruitore individuare tali peculiarità, leggere correttamente 
l'immagine e capirne la funzione nel contesto d'uso; ecco perché risulta necessario esercitarsi a tale pratica[3].

\section{Sviluppo dell'attività}

La prima fase dell'attività è stata la scelta di alcune fotografie già pubblicate nel database FOTOEDU - Archivi fotografici per la storia della scuola e dell'educazione[4], che raccoglie una parte del Fondo fotografico storico ASI (d'ora in poi ASI FF) e permette l'accesso a circa 14mila stampe scansionate e liberamente fruibili. Tale possibilità, pensata per agevolare la ricerca e il riutilizzo in ambito didattico, non limita però il riuso delle immagini digitali anche da parte di chi non si attiene alle norme di corretta citazione della fonte, facendo sì che queste, a loro volta, circolino in rete prive di riferimenti.

Abbiamo quindi svolto una ricerca avanzata sul web libero per individuare se e come tali immagini fossero state fruite e riproposte, in quali contesti e con quali caratteristiche, al fine di analizzare le tipologie di risultati che si sarebbero potute presentare. Utilizzando più motori di ricerca, si è scelto di sfruttare la funzione «per immagine» offerta da ognuno di essi, esaminando i risultati ottenuti anche attraverso te parole chiave suggerite da possibili approfondimenti d'indagine semantica.

Questo al fine di ipotizzare con quali esiti un utente del web, una volta trovata una foto attraverso la ricerca libera, possa rintracciare o avere conferma dei dati a essa relativi, citandola e utilizzandola quale fonte all'interno del proprio studio.

L'attività didattica sarà poi basata su un iter contrario a quello descritto, in cui i partecipanti sono inizialmente ignari della provenienza della fotografia. Nella sua presentazione finale l'attività si propone di:

- evidenziare i molteplici significati attribuibili alla stessa immagine a seconda dei contesti in cui essa è stata utilizzata nel web,

- porre l'attenzione sulla necessità di approfondire la correttezza delle fonti interpellate,

- sviluppare una coscienza critica nell'approccio e lettura della fotografia quale fonte, complicata dal suo essere immateriale quando digitalizzata e circolante nel web.

\section{Primo esempio}




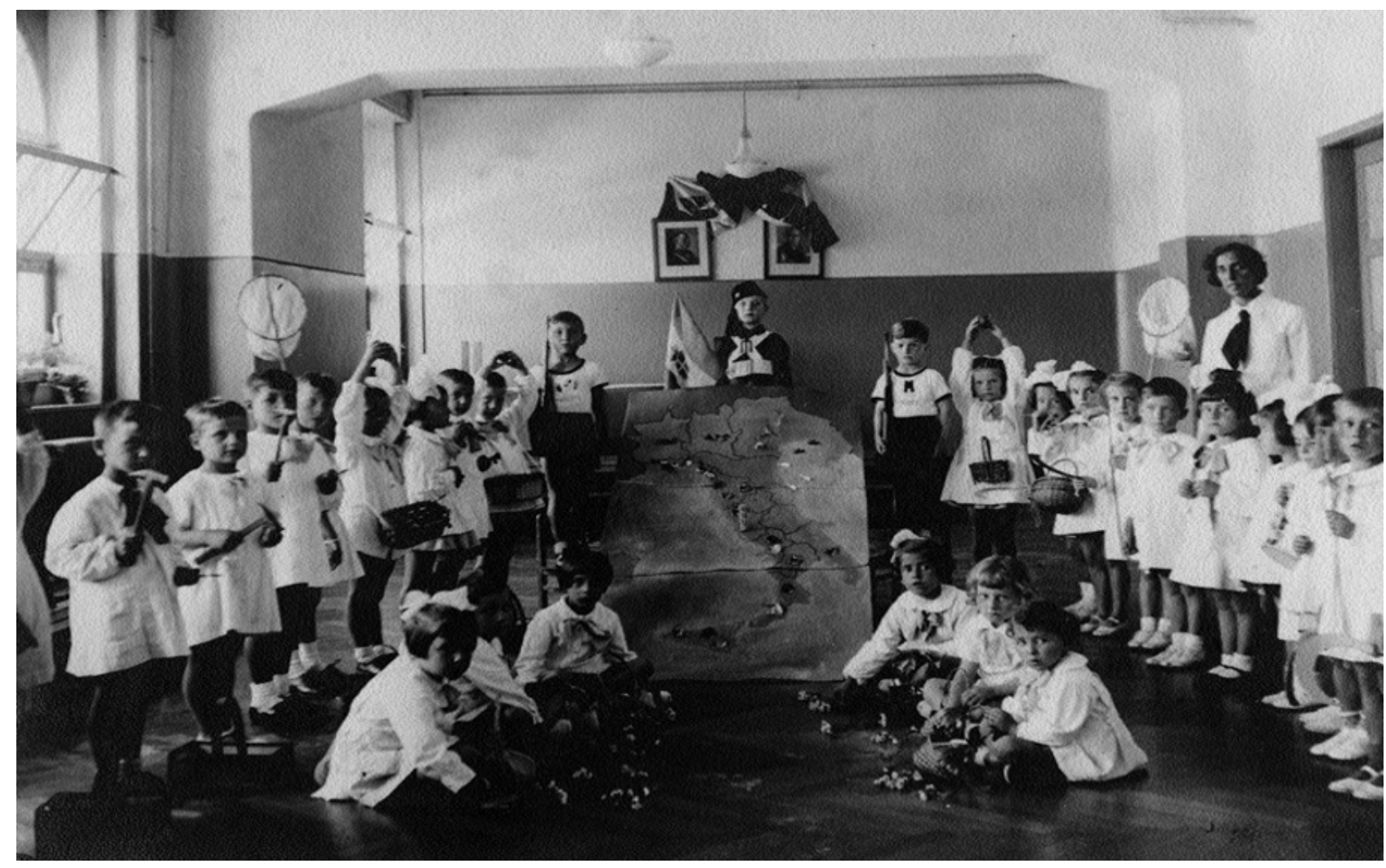

FIG.1 - La fotografia proveniente dall'Archivio Storico Indire, così come sarebbe presentata nell'ambito dell'attività didattica

Abbiamo scelto in archivio una fotografia che fosse parzialmente «facile» da leggere e collocare in ambito cronologico, anche senza conoscere altri dati se non quelli visibili nell'immagine.

Partendo anche dal fatto che l'ASI FF conserva in massima parte fotografie degli anni Quaranta, ne abbiamo selezionata una che si colloca chiaramente nel periodo fascista, riconoscibile grazie alle divise dei bambini e ai simboli alle pareti. Non presenta timbri o firme visibili sul recto. L'ambiente si può individuare come scolastico, probabilmente un'aula. Il gruppo ritratto è in posa, i bambini sono composti, ognuno di loro ha un oggetto che si presume significativo dell'attività didattica, documentata e testimoniata anche dall'elaborato posto al centro della ripresa. La fotografia è quindi «costruita», come conferma la simmetria della composizione. In questa prima breve descrizione si evidenziano aspetti che nella seconda fase dell'attività si riveleranno utili a riconsiderare i nuovi significati attribuiti alle immagini.

La scelta è caduta su questa fotografia anche per un altro aspetto: è una fotografia di memoria scolare (facilmente riconducibile a una memoria comune), è connotata da simboli politici ben identificabili, ma è anche costruita scenograficamente. Il suo essere sia documento storico, sia esteticamente significativa - "bella"- la rendono particolarmente spendibile e riutilizzabile.

Abbiamo usato lo strumento di Google per la «ricerca tramite immagini» (anche con la funzione di «ricerca inversa» o reverse image tramite url o caricamento del file jpg nel campo predisposto), arrivando a individuare tre casi particolarmente rilevanti di riuso:

\section{A - Corredo al testo}


Nel primo l'immagine è a corredo di: La donna e il fascismo[5], seconda parte di un testo già pubblicato sul sito Televignole che si definisce come «un progetto editoriale» e spazio di confronto in dialetto trentino e lingua inglese: un pezzo di stampo giornalistico quindi, benché non pubblicato su un organo di stampa riconosciuto. L'immagine, come le altre pubblicate (provenienti da più fonti, tra cui l'Archivio Luce), s'interpone a un testo che non ha rimandi diretti a esse, ed è priva di didascalia. La fotografia però ha un legame stretto con l'argomento dell'articolo,

- è chiamata a dialogare con esso per analogia tematica,

- è cronologicamente legata al momento storico,

- è inerente al tema dell'emancipazione lavorativa femminile testimoniato dall'immagine dell'insegnante nella fotografia,

- posta nel paragrafo La risposta dell'Italia, vi si lega quale rimando iconografico per la presenza della cartina geografica al centro (volendo presupporre che quest'ultima sia stata una scelta non casuale di chi ha impaginato l'intervento).

\section{B - Appendice iconografica}

In un secondo sito si riscontra un legame tra il momento storico dello scatto e il tema del post in cui è inserito dal titolo: La costruzione del consenso: il fascismo, i giovani, la famiglia[6]. Non si tratta di un testo, ma di una successione d'immagini, solo parzialmente e sinteticamente didascalizzate. La nostra fotografia, ultima della sequenza, riporta però, come tutte le altre presentate, solo la dicitura «foto dal web». Il sito che la pubblica si dichiara quale «blog collettivo» che «non rappresenta una testata giornalistica in quanto viene aggiornato senza alcuna periodicità». La data di pubblicazione coincide con il Giorno della memoria ed è, infatti, etichettato con più tag tematiche tra cui antisemitismo, Nazismo, Olocausto, ottenimento del consenso del fascismo, Shoah. Possiamo notare che la connotazione politica del sito, apertamente dichiarata nel nome e dall'url, non è rimarcata dall'aggiunta di valenza critico-interpretativa alle immagini, che quindi vengono lasciate dialogare tra loro nel richiamo di temi suggeriti dalla sequenza, il cui principale aggregatore significante è dato dal titolo del post.

\section{C - Decontestualizzata}

Infine, un terzo caso è quello in cui viene inserita in un intervento sul tema attuale del bullismo nella scuola[7]. La pagina è descritta nella home quale «sito» che tratta di storia, politica e società, mentre il taglio critico viene definito nella sezione di presentazione. Il testo non rimanda all'immagine, non la cita, essa anzi ne è una narrazione complementare e quasi autonoma. In questo caso è presente la didascalia: «Quando la scuola era ancora una cosa seria». Una frase che non è funzionale a corredarla come fonte: non si tratta intuitivamente della didascalia originaria ma le attribuisce un nuovo significato.

\section{Un'analisi critica dei risultati della ricerca}

Riassumendo, possiamo quindi rintracciare tre tipologie d'uso:

- corredo al testo,

- appendice iconografica tematica,

- decontestualizzazione 
Nella descrizione delle pagine web brevemente descritte abbiamo cercato di evidenziare le peculiarità e d'individuare in ognuna:

- la tipologia,

- la finalità,

- la connotazione.

In modo correlato possiamo anche identificare l'autorevolezza dello spazio web, quale contenitore dell'intervento in cui è inserita la fotografia in oggetto, dal carattere:

- scientifico,

- giornalistico,

- di contributo personale, soggettivo.

Come anticipato, solo nell'ultima fase dell'attività che proporremo, verrà svelata la provenienza della fotografia dall'ASI, benché non si possa escludere che l'immagine analizzata, e circolante in rete, non derivi da altre stampe dello stesso negativo. Neppure si può affermare che i dati archivistici (desunti dal catalogatore dalla copia arrivata in istituto) rispecchino le finalità originarie della fotografia. Possiamo presupporre però che raccontino comunque uno degli usi fatti nell'ambito della sua stessa committenza, l'istituto scolastico che in questa forma l'ha poi inviata al Centro Didattico Nazionale, oggi Indire.

Il riferimento è utile innanzitutto per comprendere, durante l'attività didattica, che l'immagine 'esiste' in una sua forma fisica (la stampa fotografica) con attributi materiali (formato e supporto...) e in uno spazio reale (l'archivio) e che ogni stampa rimanda a un 'originale' e ad un autore materiale della fotografia che, anche quando non risulta identificabile, rimane colui che ne ha determinato in prima istanza le caratteristiche. 


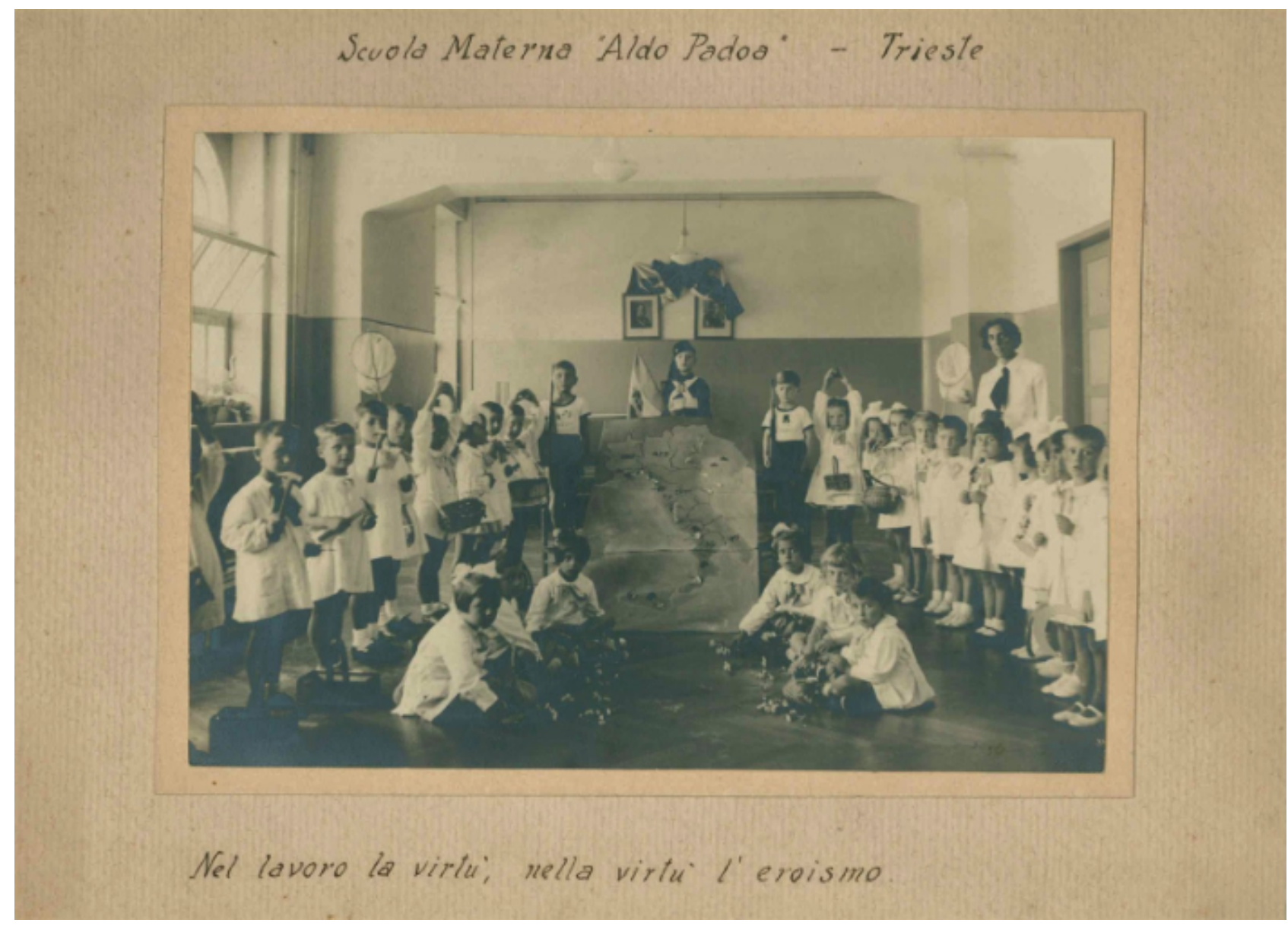

FIG. 2- La fotografia montata su cartoncino, così come si presenta in archivio Indire. ASI, Fondo fotografico, 02.311.012.

Fascismo in una scuola materna di Trieste[8]

Proveniente da: Scuola materna "Aldo Padoa", Trieste, TS, Friuli-Venezia Giulia - scuola dell'infanzia;

Fotografia Analogica 17,5x23,5 cm bianco/nero;

Soggetti: fascismo - bambino - bambina - alunno - insegnante - scuola;

didascalia originale: Scuola Materna "Aldo Padoa" - Trieste. Nel lavoro la virtù, nella virtù l'eroismo.

Categoria: Vita scolastica

Ente conservatore: INDIRE Firenze

Fondo fotografico, 02.311.012

La scheda dell'ASI rivela un titolo attribuito dall'archivista: Fascismo in una scuola materna di Trieste. Una scelta dettata dalla volontà di rendere più facilmente leggibile il database, ma anche tendenzialmente fuorviante per un lettore inesperto, al quale potrebbe apparire come la didascalia originale, indicata invece nel campo apposito[9], da cui l'archivista ha tratto le informazioni sulla provenienza. La didascalia riporta anche un contenuto connotativo-politico apposto da chi ha montato la fotografia su cartoncino, l'ha scelta e l'ha inviata all'istituto negli anni Quaranta. Ciò mostra come la fonte non sia mai neutra quanto piuttosto frutto di scelte, montaggi "significanti": 
individuarne le peculiarità è necessario per la sua corretta lettura e propedeutico al suo eventuale riutilizzo. Nella scheda non troviamo riferimenti al fotografo, neanche sul verso del documento sono rintracciabili firme o timbri. Da segnalare infine che nella versione front-end di Fotoedu sono assenti i legami tra le fotografie provenienti dalla stessa scuola presenti in archivio e nel database, probabilmente coeve e legate ad una 'serie'.

Seconda indagine

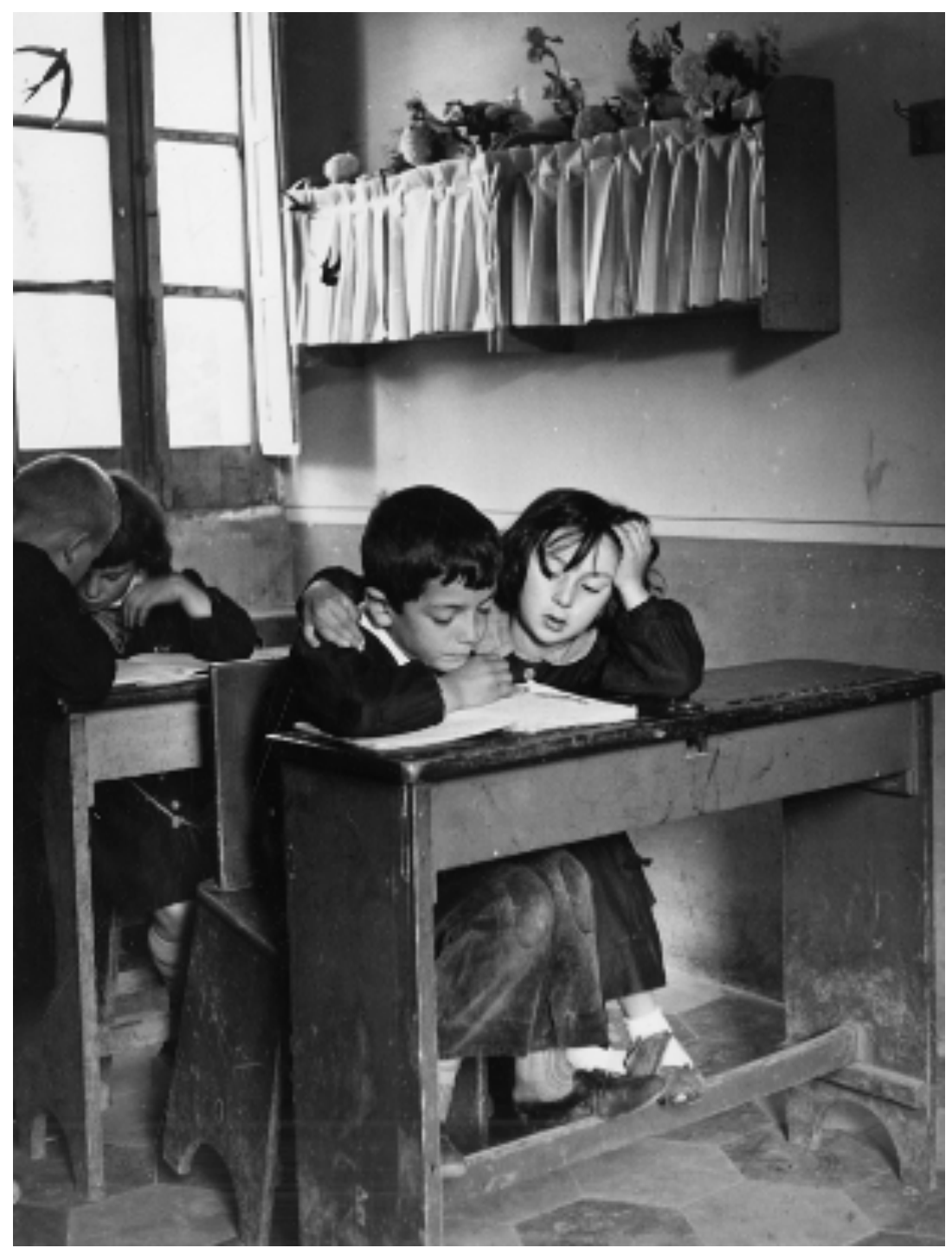

FIG. 3

Abbiamo poi provato a scegliere una fotografia, sempre dell'ASI FF, che non presentasse simboli e riferimenti politici, in modo da ipotizzare un suo riuso nel web meno connotato dal punto di vista contenutistico. Questa è catalogata come segue[10]:

Aiuto e tenerezza tra i banchi di scuola.

Data della ripresa: [s.d.] Proveniente da: Scuola elementare, Introdacqua (L'Aquila), AQ, Abruzzo - scuola primaria;

Fotografia Analogica bianco/nero;

Soggetti: bambino - bambina - banco - alunno - lettura; 
Note del contenuto: Stampa fotografica $18 \times 24 \mathrm{~cm}$, appartenente al Patrimonio fotografico dell'Indire.

Didascalia originale: "La lettura". Scuola Elementare di Noce Carlone, frazione del comune di Introdacqua (L'Aquila): tenerezza tra i banchi di scuola. Un'alunna aiuta il compagno a leggere un bel libro.

Categoria: Vita scolastica

Ente conservatore: INDIRE Firenze

Fondo fotografico, 02.053.040

La fotografia, già fornita da Indire per le copertine di due pubblicazioni di storia delle pratiche educative, risponde a esigenze sia di attendibilità documentaria, sia estetiche-evocative[11]. La si può rintracciare in diverse piattaforme social, in interventi riguardanti memorie scolastiche personali, ma anche in contesti di approfondimento storico e giornalistico con didascalie che, se presenti, non indicano la provenienza della fonte o i dati minimi per contestualizzarla[12].

Usando motori di ricerca inversa d'immagini, quali Bing Visual Search e Yandex, (motore di ricerca russo), la ritroviamo anche in molteplici profili di social network basati sulla condivisione di fotografie, quali Pinterest e Flickr, nella quasi totalità dei casi senza didascalia o alcuna connotazione testuale, tra le tante tag che la identificano ritroviamo school e vintage.

Infine, appare divertente il risultato individuato usando TinEye: reverse image search engine: una rielaborazione creativa in chiave satirica, opera di un artista che pubblica le proprie vignette su più blog e su Flickr[13]. 


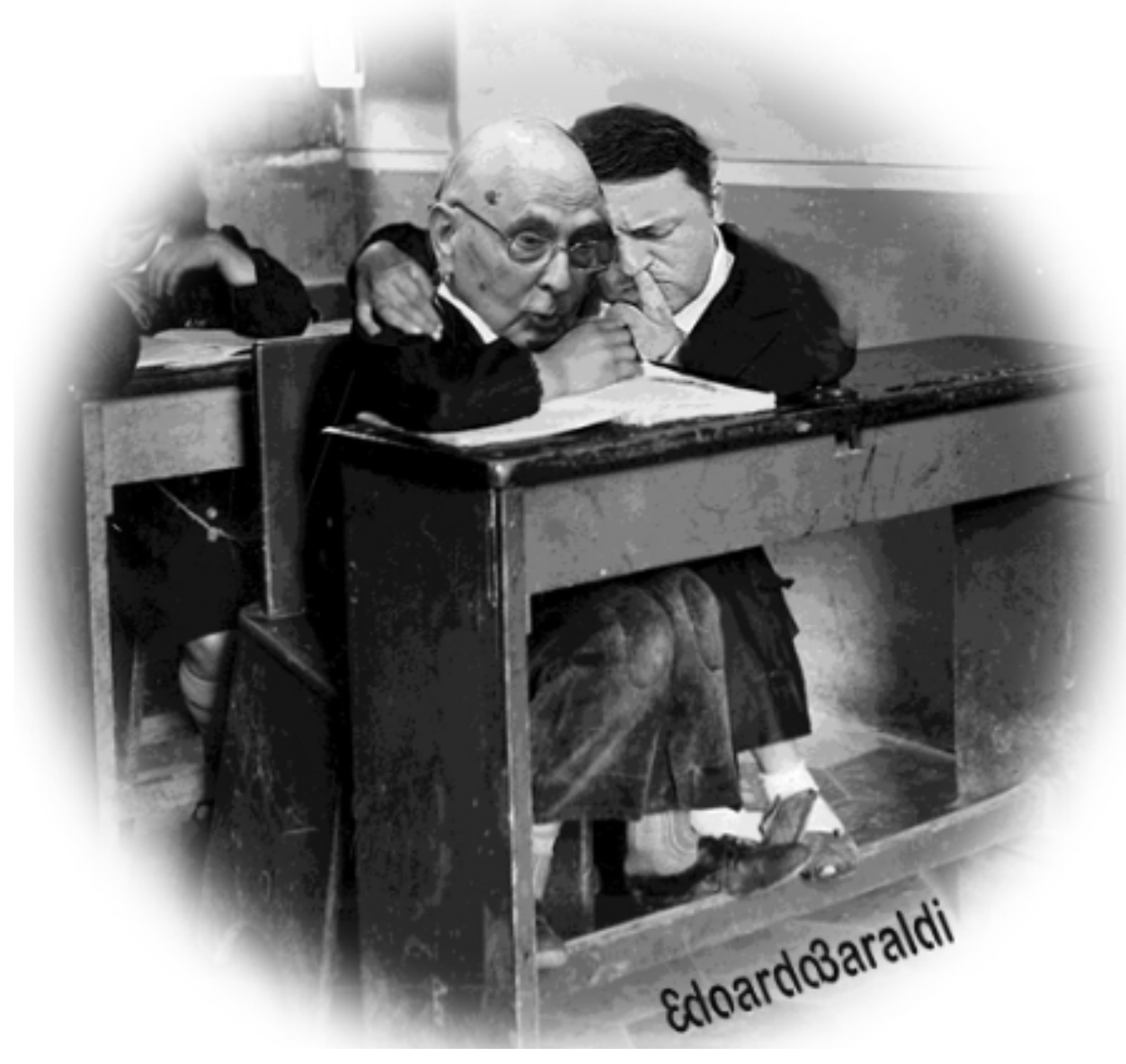

FIG. 4- La volta buona. Giorgio Napolitano: "Col No ho perso anche io. Mi dedicherò agli studi". Una «Manipolazione» di E. Baraldi, reperibile in Flickr

[https://www.flickr.com/photos/edoardobaraldi/31764330686/]

\section{Proposta per un'attività didattica}

Come abbiamo evidenziato fin qui, il web è un vero territorio da esplorare in cui, senza i giusti strumenti e un criterio con cui orientarsi, è facile 'perdersi'. L'attività che proponiamo suggerisce fasi e linee guida che non escludono risultati di volta in volta diversi e sempre stimolanti nuove discussioni.

Indicativamente questa proposta di ricerca può riguardare gli studenti e le studentesse delle scuole secondarie di secondo grado. Per realizzarla nelle scuole secondarie di primo grado sarebbe necessaria una stretta supervisione dei docenti, e che $\mathrm{i}$ bambini avessero già avuto in precedenza una formazione di base e una consuetudine di accesso controllato alla rete. 
Per quanto riguarda la scelta dei contenuti su cui esercitarsi, la corrispondenza con i temi studiati nel curricolo di storia generale in quel periodo o in quell'anno scolastico non è strettamente richiesta, poiché il lavoro si configura come laboratorio sulle fonti. È la pertinenza delle fonti che conviene privilegiare rispetto al loro legame con il periodo storico studiato.

Quanto agli strumenti, si consiglia che il docente prenda visione in anticipo delle funzioni di ricerca avanzata tramite immagine e per reverse image nei diversi motori di ricerca disponibili. I più efficaci e d'uso intuitivo che proponiamo sono i seguenti:

1) Google immagini, attivando specificatamente la funzionalità di ricerca tramite immagine ed eventualmente dei filtri.

2) TinEye;

3) Bing Visual Search;

4) Duplichecker, che come Report Seo e Hosting Checker, è un altro motore di ricerca reverse image.

La prima fase da attuare é la scelta di una fotografia presente in rete, meglio se di provenienza nota proprio per finalizzare in modo più efficace l'attività. In questo caso il docente può avvalersi di database on line a lui noti, per esempio di attinenza alla storia locale, oppure di risorse organizzate in archivi digitali quali Europeana o Fotoedu. Seppur con i limiti già evidenziati, infatti, un database organizzato resta un utile supporto per rintracciare in rete i dati minimi relativi al documento oggetto della ricerca.

Estrapolando l'immagine e presentandola ai ragazzi, si può far redigere loro, in gruppo o singolarmente, una scheda che risponda a precise domande-guida della loro analisi, chiedendo:

- una descrizione (cosa si vede, i soggetti, l'ambiente...);

- un'ipotesi su chi siano i soggetti e cosa stiano facendo;

- gli indizi individuabili nella ripresa (edifici, oggetti riconoscibili, costumi...).

Inoltre potranno avanzare ipotesi:

- sulla datazione;

- sul contesto e l'ambito in cui fu scattata la fotografia;

- $\quad$ sull'autore (se professionista/amatore, adulto/bambino, etc...);

e infine provare a riunire tali ipotesi in una breve didascalia esplicativa.

In seconda battuta, e sempre con supporto del docente, i ragazzi potranno rintracciare l'immagine sul web con uno o più dei motori di ricerca proposti, redigendo quindi una scheda dei risultati, appuntando, per ogni azione avviata:

- gli strumenti e i filtri attivati;

- $\quad$ i risultati ottenuti (link);

- una sintetica descrizione testuale in cui indicare:

1. in che tipo si sito si trova (ipotizzando le macrocategorie blog, post di un social, e articolo giornalistico o scientifico);

2. posizione e misura (centrale, a lato, icona, etc...);

3. se ha o no una didascalia/titolo e quale. 
Questo lavoro costituisce un esercizio alla lettura analitica della fotografia nel suo contesto di riuso. Dopo un confronto e una valutazione dei risultati ottenuti, i ragazzi potranno infine provare a scrivere una didascalia esplicativa della fotografia alla luce delle nuove informazioni reperite.

Solo nella parte conclusiva il docente potrà svelare i dati del documento fotografico proveniente dall' archivio e discutere analogie e differenze con i risultati delle fasi precedenti. Il processo di restituzione e di "svelamento", anche attraverso le informazioni archivistiche reperite, varrà per:

- confermare o smentire alcuni dei dati rintracciati;

- individuare traduzioni, editing e modifiche all'immagine;

- individuare i cambi di contesto rispetto a quanto ricostruito.

L'attività costituisce perciò un invito a leggere ora la fotografia in modo più critico, nei suoi ambiti di ri-uso. Infine, l'indicazione di ricerca dovrà tornare a indirizzarsi verso i contesti originari in cui la fotografia è stata prodotta, quando sia possibile individuarli. L'idea che ci sia un momento di nascita dell'immagine nella sua forma di negativo e una destinazione d'uso iniziale o comunque più antica di quelle analizzate deve rimanere ben presente nei ragazzi e nelle ragazze, tanto da invitarli da una parte a tentare di scovare la banca dati digitale o l'archivio da cui la fotografia ha iniziato il suo viaggio nel web, dall'altra a cercare informazioni sui possibili autori e loro finalità e, se noti (fotografi, ditte...), sui contesti storico-temporali e geografici in cui operarono.

\section{Conclusioni}

Questa proposta di attività offre molteplici sollecitazioni e spunti, innanzitutto in quanto, pur partendo dal mezzo digitale (che nella contingenza del confinamento da Covid-19 può rivelarsi funzionale alla didattica a distanza), si propone:

1. d'introdurre gli studenti al concetto di archivio e di documento,

2. di discutere dei concetti di attendibilità e affidabilità delle informazioni veicolate dai documenti, siano questi storici o contemporanei e reperibili nei contenuti web.

L'uso del formato digitale del documento è utile per prendere coscienza, quasi per contrasto, della necessità di considerarne la reale provenienza, la fisicità del luogo di conservazione, il processo di digitalizzazione subito e la sua materialità quale oggetto tangibile consistente di recto e verso, di un supporto primario e secondario portatore dei segni dei suoi usi sociali nel tempo quale un 'palinsesto "[14]: elementi che ci devono portare a leggerlo al di là del mero contenuto visivo. Inoltre il riuso stesso nel web dimostra come la vita della fotografia/immagine continui nel tempo, caricandosi ogni volta di nuovi significati.

Poiché ci muoviamo sempre più in un contesto di dematerializzazione dei documenti nel mondo virtuale della rete e del digitale, è importante ricordarne l'originaria coesistenza di forma e contenuto e l'esistenza di un autore e di un contesto in cui il documento fu prodotto. Tali dimensioni originarie sono precedenti a ogni successiva circolazione della fotografia/immagine, alle possibili attribuzioni di nuovi significati e decostruzioni ai fini delle molteplici narrazioni cui si presta e che abbiamo potuto incontrare con questo lavoro.

Tale attività, infatti, si configura principalmente come laboratorio sulle «fonti», e, nello specifico, l'uso di fonti fotografiche proposto è solo una delle possibili modalità per esercitarsi su questo aspetto della didattica della storia. 
In ambito didattico, infatti, il documento visivo, l'immagine fotografica più in generale, è scelto spesso poiché viene ritenuto più attraente e di più facile gestione rispetto al testo letterario $\mathrm{o} a \mathrm{l}$ documento scritto, pieni di informazioni concettuali: una facilità solo apparente, come abbiamo potuto osservare; a partire invece dalla consapevolezza della complessità di questa tipologia documentaria si possono apprezzare meglio le potenzialità del suo uso didattico.

La possibilità per gli studenti di fruire degli strumenti liberamente disponibili in rete necessita, non solo della conoscenza delle loro potenzialità, ma anche della capacità di farne un corretto utilizzo in azioni didattiche e formative finalizzate a promuovere (con il supporto dei docenti) competenze digitali non solo prettamente ricreative. La consapevolezza nell'approccio al mondo del web in generale è divenuta una necessità sempre più stringente per ogni fascia d'età, proprio in virtù della diffusione pervasiva della rete.

Acquisire consapevolezza dei suoi limiti come mezzo di ricerca e d'indagine diviene necessario quando ne vogliamo divenire fruitori responsabili, ed è un'azione che richiede un esercizio costante sempre aggiornato, come evidenzia il dibattito sulle fake news e sul bisogno di strumenti critici per l'analisi delle informazioni copiosamente disponibili on line. Una delle caratteristiche del nostro tempo è del resto data dall'uso della rete e dall'uso dei social in cui ad una massiccia circolazione delle informazioni non corrisponde facilmente la capacità di riconoscerne la genuinità $\mathrm{e}$ l'affidabilità[15].

\section{Bibliografia e fonti}

- E. Aquilini, I programmi scolastici di scienze nell'Italia unita: dal 1860 al 1955, Aracne, Ariccia 2016.

- $\quad$ G. Atzeni, Crescere, che fatica, in Le spille della prof., 23 maggio 2018, [https://lespilledellaprof.wordpress.com/2018/05/23/crescere-che-fatica/], url consultata il 31/07/2020.

- R. Barthes, La camera chiara. Nota sulla fotografia, Einaudi, Torino 2003.

- P. Bourdieu et al, La fotografia. Usi e funzioni sociali di un'arte media, Guaraldi, RiminiFirenze 1971.

- T. Serena, La profondità della superficie. Una prospettiva epistemologica per 'cose' come fotografie e archivi fotografici, in C. Caraffa, T. Serena (a cura di), Archivi fotografici. Spazi del sapere, luoghi della ricerca, numero monografico di "Ricerche di Storia dell'arte", 106, 2012, pp. 53-69.

- F. Begbie, Nostalgia del passato, reperibile in [https://it.toluna.com/opinions/2919937/Nostalgia-del-passato], url consultate il 31/07/2020.

- C. Galas, La donna e il fascismo, in Televignole, 4 marzo 2017, [http://www.televignole.it/la-donna-fascismo-2/], url consultata il 31/07/2020.

- E. Galoppini, Il "bullismo" nasce dall'assenza della famiglia e dello Stato, in Il discrimine, 2 maggio 2016, [http://www.ildiscrimine.com/il-bullismo-nasce-dallassenza-della-famigliae-dello-stato/], url consultata il 31/07/2020.

- P. Giorgi e J. Meda (a cura di), I Fondi archivistici dell'Agenzia nazionale per lo sviluppo dell'autonomia scolastica, Polistampa, Firenze 2009.

- P. Giorgi (a cura di), Dal Museo Nazionale della Scuola all'Indire. Storia di un Istituto al servizio della Scuola italiana (1929-2009), Giunti, Firenze 2010.

- O. Goti e S. Lusini (a cura di), Strategie per la fotografia. Incontro degli archivi fotografici, Archivio Fotografico Toscano, Prato, 2001. 
- M. Dal Lago, Migranti in classe,in Doppiozero, 29 aprile 2014, [https://www.doppiozero.com/materiali/sala-insegnanti/migranti-classe-gli-italianisvizzera], url consultata il 31/07/2020.

- E. Martignago, Contrappasso, in Cahier di frammenti, 2 aprile 2016, [https://medium.com/cahier-di-frammenti/000112-956afbc71ec7], url consultata il 31/07/2020.

- A. Molfese, L'analfabetismo funzionale è in rimonta?, in Gazzetta della Val d'Agri, 30 Novembre 2019, [https://www.gazzettadellavaldagri.it/lanalfabetismo-funzionale-e-inrimonta/], url consultata il 31/07/2020.

- RSF. Rivista di studi di fotografia: Fotografia e Public History, 5, 2017. Reperibile in [https://oajournals.fupress.net/index.php/rsf/issue/view/367], url consultata il 31/07/2020.

- La costruzione del consenso: il fascismo, i giovani, la famiglia, in Essere di Sinistra, 27 gennaio 2015, [https://esseresinistra.wordpress.com/tag/shoah/], url consultata il 31/07/2020.

- Progetti per la scuola, in Istituto bergamasco per la storia della Resistenza e dell'età contemporanea, s.d., [http://www.isrecbg.it/web/?page id=4436], url consultata il 31/07/2020.

- T. Serena, Per una teoria dell'archivio fotografico come "possibilità necessaria”, in G. D. Fragapane e F. Faeta (a cura di), Forme e modelli. La fotografia come modo di conoscenza, Atti del convegno (Noto, 2010), Corisco Cosenza. Reperibile in [http://www.coriscoedizioni.it/wp-content/uploads/2013/10/Forme-e-Modelli.-Lafotografia-come-modo-di-conoscenza.pdf], url consultata il 31/07/2020.

- S. Sontag, Sulla fotografia. Realtà e immagine nella nostra società, Einaudi, Torino 2004.

- N. Tiretta, Ricordi: Le scuole a Montella negli anni 45/50/60, in Montella.eu, 14 marzo 2019, [https://www.montella.eu/i-vostri-articoli/44-arte-e-cultura/2222-ricordi-le-scuole-amontella-negli-anni-45-50-60], url consultata il 31/07/2020.

- L. Vanni, Per un'archeologia della scuola: le lunghe durate e il palinsesto, CLUEB, Bologna 2011.

- F. Zanetti, Facciamoci due risate: la scuola vintage, dal blog Dannata Vintage, 13 febbraio 2018, [https:/www.dannatavintage.com/2018/02/13/la-scuola-vintage/], url consultata il 31/07/2020.

- I. Zoppi, Gli album fotografici dell'Archivio Storico Indire. Memorie scolastiche degli anni Quaranta tra esposizione e archiviazione, in "RSF. Rivista di studi di fotografia", 4, 2016, pp. 88-99. Reperibile in

[https://oajournals.fupress.net/index.php/rsf/article/view/8880/8878], url consultata il $31 / 07 / 2020$.

Sitografia

- FOTOEDU-Archivi fotografici per la storia della scuola e dell'educazione, [http://www.indire.it/progetto/fotoedu/], url consultata il 31/07/2020.

- E. Baraldi, Inclusioni e manipolazioni, [http://www.edoardobaraldi.it/inclusioni_manipolazioni1.htm], url consultata il 31/07/2020.

\section{Documenti d'archivio}

- ASI, Fondo fotografico, 02.311.012.

- ASI, Fondo fotografico, 02.053.040. 
Note:

[1] Tra cui quelli svolti nell'ambito di Lucca Internet Festival 2018, Didacta 2018 e 2019.

[2] Per la storia dei fondi storici Indire e in particolare del fondo fotografico cfr. P. Giorgi e J. Meda (a cura di), I Fondi archivistici dell'Agenzia nazionale per lo sviluppo dell'autonomia scolastica, Polistampa, Firenze 2009; P. Giorgi (a cura di), Dal Museo Nazionale della Scuola all'Indire. Storia di un Istituto al servizio della Scuola italiana (1929-2009), Giunti, Firenze 2010. I. Zoppi, Gli album fotografici dell'Archivio Storico Indire. Memorie scolastiche degli anni Quaranta tra esposizione e archiviazione, in "RSF. Rivista di studi di fotografia", 4, 2016, pp. 88-99. Reperibile in [https://oajournals.fupress.net/index.php/rsf/article/view/8880/8878], url consultata il $31 / 07 / 2020$.

[3] Riguardo all'analisi della fotografia come fonte storica cfr. A. Mignemi, Lo sguardo e l'immagine. La fotografia come documento storico, Bollati Boringhieri, Torino, 2003.

[4] FOTOEDU - Archivi fotografici per la storia della scuola e dell'educazione, [http://www.indire.it/progetto/fotoedu/], url consultata il 31/07/2020.

[5] C. Galas, La donna e il fascismo, in Televignole, 4 marzo 2017, [http://www.televignole.it/ladonna-fascismo-2/], url consultata il 31/07/2020.

[6] S.a, La costruzione del consenso: il fascismo, i giovani, la famiglia, in Essere di Sinistra, 27 gennaio 2015, [https://esseresinistra.wordpress.com/tag/shoah/], url consultata il 31/07/2020.

[7] E. Galoppini, Il "bullismo" nasce dall'assenza della famiglia e dello Stato, in Il discrimine, 2 maggio 2016, [http://www.ildiscrimine.com/il-bullismo-nasce-dallassenza-della-famiglia-e-dellostato/], url consultata il 31/07/2020.

[8] Scheda catalografica ASI FF, reperibile in fotoeducatalogo.indire.it.

[9] Questo ci porta anche a ricordare come gli stessi database accessibili in rete non seguano standard descrittivi uniformi, e seguano scelte dettate da necessità diversificate. La fruizione e l'accesso completo alle informazioni diventa quindi non sempre facile o possibile, specie ad un utente non esperto.

[10] Scheda catalografica ASI, reperibile in fotoeducatalogo.indire.it.

[11] La fotografia è stata utilizzata per le copertine dei volumi L. Vanni, Per un'archeologia della scuola: le lunghe durate e il palinsesto, CLUEB, Bologna 2011; E. Aquilini, I programmi scolastici di scienze nell'Italia unita: dal 1860 al 1955, Aracne, Ariccia 2016.

[12] Si riportano alcuni esempi: F. Zanetti, Facciamoci due risate: la scuola vintage, dal blog Dannata Vintage, 13 febbraio 2018, [https://www.dannatavintage.com/2018/02/13/la-scuolavintage/], url consultata il 31/07/2020.

N. Tiretta, Ricordi: Le scuole a Montella negli anni 45/50/60, in Montella.eu, 14 marzo 2019, [https://www.montella.eu/i-vostri-articoli/44-arte-e-cultura/2222-ricordi-le-scuole-a-montella-neglianni-45-50-60], url consultata il 31/07/2020. FrancoBegbie, Nostalgia del passato, in Toluna, s.d., [https://it.toluna.com/opinions/2919937/Nostalgia-del-passato], url consultata il 31/07/2020.

G.Atzeni, Crescere, che fatica, in Le spille della prof., 23 maggio 2018,

[https://lespilledellaprof.wordpress.com/2018/05/23/crescere-che-fatica/], url consultata il 
31/07/2020. A. Molfese, L'analfabetismo funzionale è in rimonta?, in Gazzetta della Val d'Agri, 30 Novembre 2019, [https://www.gazzettadellavaldagri.it/lanalfabetismo-funzionale-e-in-rimonta/], url consultata il 31/07/2020, S.a., Progetti per la scuola, in Istituto bergamasco per la storia della Resistenza e dell'età contemporanea, s.d., [http://www.isrecbg.it/web/?page_id=4436], url consultata il 31/07/2020.

Unica eccezione che riporta i crediti fotografici relativi a Indire, pur senza didascalia è in M. Dal Lago, Migranti in classe, in Doppiozero, 29 aprile 2014, [https://www.doppiozero.com/materiali/sala-insegnanti/migranti-classe-gli-italiani-svizzera], url consultata il 31/07/2020. L'articolo è una recensione critica dell'omologo studio e pubblicazione di P. Barcella, Migranti in classe: gli Italiani in Svizzera tra scuola e formazione professionale, Ombre corte, Verona 2014.

[13] E. Baraldi, Inclusioni e manipolazioni,

[http://www.edoardobaraldi.it/inclusioni_manipolazioni1.htm], url consultata il 31/07/2020.

[14] Cfr. T. Serena, La profondità della superficie. Una prospettiva epistemologica per 'cose' come fotografie e archivi fotografici, in C. Caraffa, T. Serena (a cura di), Archivi fotografici. Spazi del sapere, luoghi della ricerca, numero monografico di "Ricerche di Storia dell'arte", 106, 2012, pp. 53-69.

[15] Sul tema si rimanda al recente, breve quanto esauriente, intervento di Linda Giuva Gli archivi al tempo delle fake news, nell' ambito del ciclo di incontri Gli Archivi. Roba che si mangia?, organizzato da Archivistica Attiva (interventi on line, 7 aprile 2020), [https://youtu.be/SWamMEn8WzI], url consultata il 31/07/2020. 